\title{
The impact of obesity and timely antiviral administration on severe influenza outcomes among hospitalized adults
}

\author{
Hannah E. Segaloff ${ }^{1}$ (iD $\mid$ Richard Evans $^{1}$ | Samia Arshad ${ }^{2}$ | Marcus J. Zervos ${ }^{2}$ \\ Carolyn Archer $^{3}$ | Keith S. Kaye ${ }^{4}$ | Emily T. Martin ${ }^{1}$
}

${ }^{1}$ Department of Epidemiology, University of Michigan School of Public Health, Ann Arbor, Michigan

2 Division of Infectious Diseases, Henry Ford Health System, Detroit, Michigan

${ }^{3}$ Department of Pharmacy Practice, Wayne State University, Detroit, Michigan

${ }^{4}$ Division of Infectious Diseases, Department of Internal Medicine, University of Michigan Health System, Ann Arbor, Michigan

\section{Correspondence}

Hannah Segaloff, Department of

Epidemiology, University of Michigan School of Public Health, 1415 Washington Heights, Ann Arbor, MI 48109.

Email: segaloff@umich.edu

Funding information

American Association of Colleges of Pharmacy
Obesity was identified as a risk factor for severe influenza during the 2009 influenza A(H1N1)pandemic, but evidence of this association has been mixed since. Postpandemic antiviral treatment guidelines may have increased antiviral treatment among obese individuals. A prospective study of adults hospitalized with laboratoryconfirmed influenza in Detroit, Michigan in 2011-2012 and 2012-2013 was conducted. Patient information was collected from interviews and medical chart abstraction. Obese $(\mathrm{BMI} \geq 30)$ and non-obese $(\mathrm{BMI}<30)$ participants were compared. Late antiviral treatment ( $>2$ days from symptom onset), obesity $(30 \leq \mathrm{BMI}<40)$, and morbid obesity $(B M I \geq 40)$ were evaluated as predictors of lower respiratory tract disease (LRD), ICU admission, and length of stay (LOS) using logistic regression and inverse probability weighted models. Forty-eight participants were included in the study after exclusions and all patients received antiviral treatment. Participants who were obese were significantly more likely to have a cough and to take steroids than non-obese participants, and had a shorter time from hospital admission to antiviral treatment (median time from admission to treatment of 0 days for obese patients and 1 day for non-obese patients $[P=0.001])$. In all models, late antiviral treatment was associated with increased odds of LRD (OR: 3.9 [1.1,15.9] in fully adjusted model). After adjustment for treatment timing, the odds of ICU admission (OR: 6.4 [0.8,58.2] to 7.9 $[0.9,87.1])$ and LRD (OR: $3.3[0.5,23.5]$ to $4.0[0.6,35.0]$ ) associated with morbid obesity increased. Obese individuals were treated with antivirals earlier than others. Late antiviral treatment was associated with severe influenza in the hospital.

\section{KEYWORDS}

antiviral agents, epidemiology, influenza virus, neuraminidase inhibitor

\section{1 | INTRODUCTION}

Influenza virus, though usually a self-limiting infection, is associated with increased morbidity and mortality during annual outbreaks. ${ }^{1}$ In

Institution: Wayne State University, Detroit Medical Center, Henry Ford Hospital, and University of Michigan. general, older adults and individuals with underlying comorbid conditions are at high risk for adverse events after influenza infection. ${ }^{2}$ In 2009, the emergence of the 2009 pandemic influenza A(H1N1) virus lead to an increased prevalence of severe outcomes among populations that had not previously been considered at high risk for these consequences of disease. Specifically, children and young adults were more likely to be hospitalized for influenza; and morbid obesity 
(body mass index of 40 or greater) was identified as a predictor of hospitalization, ICU admission, and of death. ${ }^{3-5}$

Subsequent analyses using data from non-pandemic influenza seasons have left an unresolved picture of the relationship between obesity and influenza severity and whether this relationship persists beyond the influenza $\mathrm{A}(\mathrm{H} 1 \mathrm{~N} 1)$ pdm09 subtype. A study by Cocoros et al. regarding seasonal and pandemic influenza $A(H 1 N 1)$ found that obesity had a small association with severe influenza like illness (ILI) in seasons dominated by H1N1 before and after the 2009 pandemic, leading to hospitalization. ${ }^{6}$ However, these results were only seen in certain age groups in the study population and these results have not been consistently validated in other populations. ${ }^{7,8}$

Differences in antiviral treatment timing add further complexity to this issue in the post-pandemic period. A meta-analysis analyzing the relationship between obesity and influenza $A(H 1 N 1) p d m 09$ severity in 2009-2011 influenza seasons globally found that the significant relationship between obesity and influenza complications was attenuated and non-significant after adjustment for antiviral prescription timing; the authors found that obese individuals were less likely to receive antivirals in a timely fashion and that this treatment timing was an important confounder of the relationship between obesity and influenza severity. ${ }^{9}$ However, in the United States, a recent change to antiviral treatment recommendations may have altered this relationship between treatment timing and weight status. After review of data from the 2009 pandemic, the Center for Disease Control and Prevention (CDC) added morbid obesity to their list of indicators for high influenza severity risk and recommended that these individuals be prescribed antiviral treatment empirically in the outpatient setting. ${ }^{10}$ These recommendations may lead physicians to treat hospitalized obese patients earlier than patients without a high-risk condition, either through empiric treatment in outpatient settings before presentation to the hospital, or through immediate treatment upon hospital admission. Previous studies have demonstrated that treatment with oseltamivir within $48 \mathrm{~h}$ of symptom onset reduces severe complications of influenza and that oseltamivir is most effective when given within $48 \mathrm{~h} .{ }^{11,12}$ However, oseltamivir is also associated with reduced duration of shedding when it is given within $72 \mathrm{~h}$ of symptom onset, and increased survival and decreased severity for up to 5 days after illness onset. ${ }^{13-15}$ If obese individuals are treated earlier than others, antiviral receipt timing may complicate the ability to detect an association between obesity and influenza severity.

In order to evaluate the relationship between antiviral timing, obesity, and influenza severity, a prospective study of hospitalized influenza-positive adults in Detroit, Michigan was conducted. A previous study in this region found that nearly $50 \%$ of the study population had a body mass index (BMI) classified as obese, well over the state average of $30.7 \% .{ }^{16}$ The results of this study indicated that obese individuals were more likely than non-obese individuals to be admitted to inpatient care, to have hospital stays of greater than 7 days, and to have lower respiratory tract disease manifestations, following influenza infection, predominately with influenza $A(H 1 N 1)$ pdm09. ${ }^{17}$ The aim of this current study is to use a prospective design and data from the 2011 to 2012 and 2012 to 2013 influenza seasons to expand on the previous data linking obesity to influenza severity, and to evaluate the role of the timing of antiviral administration in this association.

\section{2 | METHODS}

\subsection{Study population}

Hospitalized adults admitted to one of five Detroit, Michigan area hospitals with laboratory confirmed influenza from February through April 2012 and November 2012 through March 2013 were prospectively identified. Participants were enrolled from Detroit Receiving Hospital, Harper Hospital, Hutzel Women's Hospital, Sinai-Grace Hospital, and Henry Ford Hospital. Patients were identified from a clinical microbiology laboratory results using a clinical alert system (Theradoc) and their eligibility status was confirmed with their treating physician. Patients admitted to the hospital were eligible if they were 18 years old or greater, and if they tested positive for influenza A (H1N1)pdm09, influenza A (H3N2), or influenza $B$, and if they had any symptom compatible with influenza like illness (ILI) including cough, chills, rhinorrhea, myalgia, dyspnea, diarrhea, vomiting, and/or subjective fever. Patients were excluded from the analysis if they had symptom duration longer than 10 days before admission and if they had a BMI less than 18.5. Eligible patients were approached for informed consent.

\section{2 | Participant survey and data abstraction}

After affirmative consent, patients were surveyed to determine their illness onset date, physical characteristics, alcohol and smoking histories, living situation, and influenza and pneumococcal vaccine information. There were also asked about any physical, mental, or emotional limitations and if they routinely used special equipment due to a health problem (i.e. wheelchair, cane, special bed, special telephone). All day 1 interview questions were adapted from the Centers for Disease Control and Prevention Behavioral Risk Factor Surveillance System Survey Questionnaire. ${ }^{18}$ Additional information regarding demographics, insurance information, and medical information pertaining to symptoms, admission timing, antiviral and antibiotic therapy, vaccination, readmission, ICU admission, laboratory results, virus testing results, diagnoses, and comorbidities was collected from the electronic medical record (EMR). Specific conditions evaluated were a history of cancer, lung disease (bronchiectasis, COPD/ emphysema, asthma, restrictive disease, interstitial lung disease), history of heart conditions (myocardial infarction, coronary artery disease, coronary artery bypass grafting), diabetes, renal disease, and HIV. Steroid use, including use of glucocorticoid steroids, prednisone, or methylprednisolone in the last month, was also abstracted from the EMR. The patients were interviewed again by phone 30 days after enrollment to collect information on any new hospital admissions and the reason for these visits as well as any visit to a doctor in a doctor's office and the reason for these visits. Information on medications prescribed since discharge was also collected and patients were asked 
whether they had made diet or exercise changes since their discharge date.

\section{3 | Statistical analysis}

Severe outcomes in this study were defined as ICU admission, lower respiratory tract disease (intubation, hypoxia, lung infiltrates, or consolidation) and increased length of stay. For the determination of lower respiratory tract disease, hypoxia was defined as oxygen saturation percentage marked as abnormal (below 94\%), and lung infiltrates as well as consolidation were defined by a description of infiltrates or consolidation on chest X-ray impressions. In frequency models, obese (BMI of 30 or greater) and non-obese (BMI less than 30 ) participants were compared; $P$ values were determined using Fisher's exact tests. Differences in distributions of continuous variables were tested using the Wilcoxon rank-sum test. Unadjusted and adjusted Firth-penalized logistic models were run to predict odds of severity; unadjusted models contain either categorical BMI (BMI less than 30, $\mathrm{BMI}$ of 30 to less than 40 , BMI of 40 or greater) or dichotomized antiviral treatment timing (late treatment defined as $>2$ days from symptom onset), adjusted models contained both variables. Inverse probability weighting (IPW) of propensity scores was used to efficiently adjust for age, diabetes, and poor/fair self rated health despite the small sample size. Diabetes was deemed the most important clinical confounder due to the impact of immune system disruption on influenza outcomes and to the recommendations that antivirals be used promptly in these patients. ${ }^{19,20}$ Asthma was not included in the model because it did not improve model fit. IPW of propensity scores was used to reduce bias in effect estimates by balancing the baseline characteristics between those with and without the outcome of interest. Due to collinearity between steroid use and other elements in the model it was not used as an adjustment factor. For the outcome of increased length of stay, length of stay was logtransformed and linear regression was used to predict percentage change in length of stay. All statistics were run on SAS 9.4 (Statistical Analysis System).

\section{3 | RESULTS}

There were 55 individuals enrolled in this study with laboratoryconfirmed influenza. Individuals were excluded if they had missing data on BMI, lower respiratory tract disease, or ICU admission. Additionally, individuals who reported symptom onset of greater than 10 days before admission were excluded. The final analysis was performed on the 48 individuals with complete data. All $(N=48)$ participants were treated with oseltamivir, three of these patients were given oseltamivir 1 day before their study admission. Of these 48 participants, 34 (70.8\%) completed the 30-day follow up survey.

Twenty-four (50\%) participants were obese and five (10.4\%) were morbidly obese. The median age was 54.5 years of age in obese individuals and 60.5 years of age in non-obese individuals. Obese individuals had significantly shorter duration from admission to antiviral therapy and significantly greater frequency of steroid prescription in the prior three months (Table 1). At study enrollment, approximately $50 \%(n=23)$ of participants self-reported the need for special equipment due to a health problem, almost $(n=28) 60 \%$ reported having physical, mental, or emotional limitations, over $50 \%$ $(n=25)$ reported poor/fair health, and nearly $80 \%(n=37)$ reported having shortness of breath that affects their quality of life. All of these self-reported health conditions were more common among obese patients, though these differences were not statistically significant (Table 2). Fifty percent $(n=24)$ of participants reported receiving an influenza vaccine in the last year and $62.5 \%(n=30)$ reported receiving a pneumococcal vaccine during their lifetime (Table 2).

Thirty-four individuals participated in the survey given approximately 1 month post-discharge. 79.4\% ( $n=26)$ of individuals reported having an appointment with their primary care physician, 15 (57.7\%) of these appointments were follow up appointments and $20.5 \%(n=7)$ reported being readmitted to the hospital within 30-days of hospital discharge. Reasons for readmission included deep vein thrombosis, chest pain and coronary artery disease, pneumonia, ischemic cardiopathy, chronic obstructive pulmonary disease exacerbation, and congestive heart failure exacerbation. Almost $70 \%(n=26)$ of participants reported receiving a new prescription at discharge and nearly $70 \%(n=17)$ of those who received a prescription completed the full dose of the new medication. A variety of medications were prescribed in the 30 days post-discharge including antibiotics, antivirals, steroids (inhaled and ingested), blood pressure medication, pain medication, and blood thinners, among others. Over half $(52.9 \%$, $n=18$ ) of participants reported making a positive change to their diet or exercise habits 30-days after hospital discharge (Table 3). There were no deaths within 30 days of discharge.

In univariate models, late antiviral treatment was significantly associated with increased odds of lower respiratory tract disease and increased length of hospitalization (OR: 3.6 [1.1, 14.2], Percent change: 40.8 [2.6, 93.2]) (Table 4). After adjustment for BMI group, the odds of lower respiratory tract disease and ICU admission associated with late antiviral treatment increased in magnitude. In the IPW models the association between late antiviral treatment and lower respiratory tract disease remained significant (OR: 3.9 [1.1, 15.9]).

In the univariate model, obese individuals (BMI from 30 to less than 40) had significantly shorter length of hospitalization than nonobese individuals (Percent change: -29.2 [-49.6, -0.6]) (Table 4). Obesity and morbid obesity were also associated with increased odds of ICU admission and lower respiratory tract disease, and these relationships were monotonic, though not significant (Table 4). After adjustment for late-antiviral treatment the odds of lower respiratory tract disease and ICU admission increased in the obese group, though they did not reach statistical significance.

\section{4 | DISCUSSION}

This study demonstrated that antiviral treatment within two days of symptom onset was associated with reduced odds of lower respiratory 
TABLE 1 Patient characteristics by obesity status

\begin{tabular}{|c|c|c|c|c|}
\hline Characteristics & Total $(N=48)$ & $\mathrm{BMI} \geq 30(N=24)$ & $\mathrm{BMI}<30(\mathrm{~N}=24)$ & $P$-value* \\
\hline Age (median, range) & $59.0(21-91)$ & $54.5(21-83)$ & $60.5(21-91)$ & 0.30 \\
\hline BMI (mean, 95\%Cl) & $30.3(27.6,33.0)$ & - & - & - \\
\hline $\begin{array}{l}\text { Days from admission to antiviral } \\
\text { treatment (median, range }{ }^{a} \text { ) }\end{array}$ & $N(\%$ of total) & $N$ (\% of obese) & $N(\%$ of not obese) & $P$-value** \\
\hline White & $5(10.6)$ & $2(8.7)$ & $3(12.5)$ & \\
\hline Black & $39(83.0)$ & $21(91.3)$ & $18(75.0)$ & \\
\hline Other & $3(6.4)$ & $0(0.0)$ & $2(8.3)$ & \\
\hline \multicolumn{5}{|l|}{ Symptoms } \\
\hline Chills & $22(45.8)$ & $13(54.2)$ & $9(37.5)$ & 0.25 \\
\hline Shortness of breath & $29(60.4)$ & $17(70.8)$ & $12(50.0)$ & 0.14 \\
\hline Fatigue & $12(25.0)$ & $4(16.7)$ & $8(33.3)$ & 0.18 \\
\hline \multicolumn{5}{|l|}{ Clinical factors } \\
\hline Diabetes & $16(31.2)$ & $8(33.3)$ & $7(29.2)$ & 0.76 \\
\hline Chronic obstructive pulmonary disease & $11(22.9)$ & $7(29.2)$ & $4(16.7)$ & 0.30 \\
\hline Asthma & $8(16.7)$ & $5(20.8)$ & $3(12.5)$ & 0.44 \\
\hline Congestive heart failure & $11(22.9)$ & $5(20.8)$ & $6(25.0)$ & 0.73 \\
\hline
\end{tabular}

${ }^{a}$ Three obese patients were given oseltamivir 1 day before admission, these individuals were classified as treated 0 days from hospital admission. bone individual with obesity is missing race status.

'One individual with obesity is missing information on steroid medication.

${ }^{*} P$ values are the result of Wilcoxon tests.

${ }^{* *} P$ values are the result of Fisher's exact tests.

TABLE 2 Day 1 survey results by obesity status

\begin{tabular}{|c|c|c|c|c|}
\hline & Total $(N=48)$ & $\mathrm{BMI} \geq 30(\mathrm{~N}=24)$ & $\mathrm{BMI}<30(\mathrm{~N}=24)$ & \\
\hline Self-reported limitations & $28(58.3)$ & $16(66.7)$ & $12(50.0)$ & 0.24 \\
\hline Self-reported poor/fair health & $25(52.1)$ & $15(62.5)$ & $10(41.7)$ & 0.24 \\
\hline Self-reported need for equipment due to medical condition & $23(47.9)$ & $13(54.2)$ & $10(41.7)$ & 0.39 \\
\hline Report that shortness of breath affects quality of life & $37(77.0)$ & $20(83.3)$ & $17(70.8)$ & 0.30 \\
\hline Influenza vaccine receipt & $24(50.0)$ & $13(54.2)$ & $11(45.8)$ & 0.56 \\
\hline \multirow[t]{2}{*}{ Pneumonia vaccine receipt } & $30(62.5)$ & $14(58.3)$ & $16(66.7)$ & 0.55 \\
\hline & Median (range) & Median (range) & Median (range) & $P$-value** \\
\hline Alcoholic drinks per day in past month & $0(0-5)$ & $0(0-4)$ & $0(0-5)$ & 0.22 \\
\hline
\end{tabular}

${ }^{*} P$ values are the result of Fisher's exact tests.

${ }^{* *} P$ values are the result of Wilcoxon tests. 
TABLE 3 Day 30 survey results by obesity status

\begin{tabular}{|c|c|c|c|c|}
\hline Characteristics & Total $(N=34)(\%)$ & $\mathrm{BMI} \geq 30(\mathrm{~N}=20)(\%)$ & $\mathrm{BMI}<30(\mathrm{~N}=14)(\%)$ & $P$-value* \\
\hline Hospital readmission & 7 (20.6) & $3(15.0)$ & $4(28.6)$ & 0.41 \\
\hline Visit to PCP & $26(79.4)$ & $16(80.0)$ & $10(71.4)$ & 0.69 \\
\hline New medication prescribed ${ }^{a}$ & $26(67.6)$ & $14(73.7)$ & $12(85.7)$ & 0.67 \\
\hline Completed dose of new medication ${ }^{b}$ & $17(68.0)$ & $11(84.6)$ & $6(50.0)$ & 0.10 \\
\hline
\end{tabular}

${ }^{a}$ One individual with obesity is missing information on new medication prescription.

${ }^{b}$ Percentages are calculated out of the number of individuals who reported having a new medication prescribed. One obese individual who reported receiving a new prescription had missing information on dose, so this percentage was calculated out of 13 .

${ }^{*} P$ values are calculated from Fisher's exact tests.

tract disease among adults hospitalized with laboratory-confirmed influenza. In 2011, the Advisory Committee on Immunization Practices (ACIP) released their updated recommendations for antiviral treatment. They recommended that all hospitalized patients with suspected influenza be treated empirically, even before confirmation of influenza status. ${ }^{10}$ The results of this study add to the existing literature that support this treatment recommendation change by emphasizing the importance of early antiviral treatment on improving patient outcomes. $^{21,22}$ Late antiviral treatment also appears to be an important confounder between obesity and influenza severity, as the likelihood of severe disease increased among obese individuals after adjustment for treatment in all models. These findings add to previous observations of the connection between antiviral treatment and obesity; a 2016 paper examined antiviral treatment among hospitalized patients from 2010 to 2015 and found that individuals with high risk conditions, including morbid obesity, were significantly more likely than non-obese individuals to receive antivirals; however, the 2016 study did not evaluate timing of therapy. ${ }^{23}$

This study took place at hospitals in Detroit, Michigan, a city with high levels of obesity and poverty. ${ }^{24}$ As expected in hospitals in an underserved area, many participants had self-reported poor health in addition to the need for special medical equipment and physical, mental, or emotional limitations. Forty-six percent of patients received health insurance from Medicaid or a Medicare/Medicaid combination

TABLE 4 Predictive models of influenza severity among hospitalized adults treated with antivirals

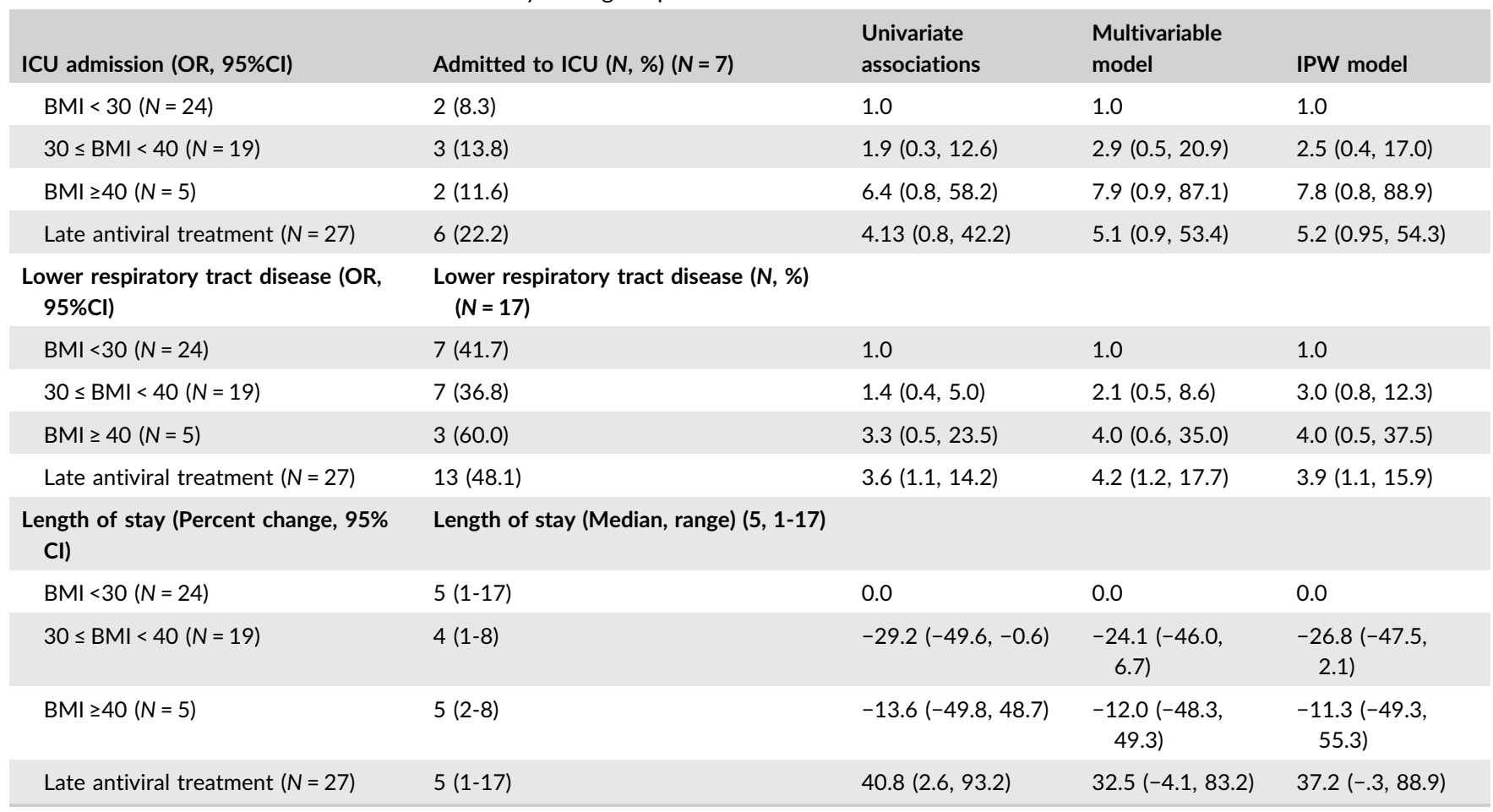

Univariate associations model displays Firth corrected univariate associations. Multivariable model displays Firth corrected associations adjusted for BMI and late antiviral treatment (treatment $>2$ days from symptom onset compared to treatment $\leq 2$ days from onset). IPW model displays inverse probability weighted models adjusted for age, diabetes and poor/fair self-rated health. Length of stay models were modeled as log (length of stay); results were transformed to display percent changes. 
and an additional $4 \%$ had no insurance. Individuals with public insurance are more likely to visit the emergency department over their primary care physician. It is unclear whether or not this is due to decreased access to their primary care physician, more complex conditions that require emergency facilities, or a preference for the hospital over outpatient clinics. ${ }^{25,26}$ In the current study, the increased use of the emergency room among those with public insurance is reflected in the high readmission rate $(20.6 \%)$ reported from respondents to the 30-day survey. Encouragingly, 52.9\% of participants who completed the 30-day survey reported efforts to improve their diet or exercise routine post-hospitalization, indicating that the hospital discharge may have the potential to be an effective time to counsel the patient on modifiable health behaviors.

There are limitations to this analysis. The in-hospital observational nature of the study complicated our ability to study some commonly used severity endpoints. Reverse causation could have masked associations between timely antiviral treatment and severe outcomes if individuals were treated when admitted to the ICU or if lower respiratory tract disease was already present at the time of treatment. Lower than expected enrollment, particularly during the mild 20112012 season, presented numerous difficulties. Though steroid use was likely an important confounder of obesity and severe influenza, steroid use could not be adjusted for due to collinearity between steroids and other adjustment factors. A variety of other confounders were able to be adjusted for using inverse-probability weighting despite the small sample size; however, it is possible that residual confounding remains. Future studies should be conducted to re-evaluate the associations studied in this analysis, as the small sample size led to a reduction in statistical power.

The findings of this prospective study highlight the need to evaluate confounding from antiviral treatment timing when studying the relationship between obesity and influenza severity. Additionally, the association between late antiviral treatment and increased likelihood of serious disease highlights the importance of timely antiviral treatment. This reinforces the treatment recommendations from the ACIP and emphasizes the need for continued evaluation of antiviral prescription rates among hospitalized adults.

\section{ORCID}

Hannah E. Segaloff (iD http://orcid.org/0000-0003-4716-7468

\section{REFERENCES}

1. Simonsen L, Clarke MJ, Williamson GD, Stroup DF, Arden NH, Schonberger LB. The impact of influenza epidemics on mortality: introducing a severity index. Am J Public Health. 1997;87: 1944-1950.

2. Centers for disease control and prevention. estimates of deaths associated with seasonal influenza-United States, 1976-2007. MMWR. 2010;59:1057.

3. van Kerkhove MD, Vandemaele $\mathrm{KAH}$, Shinde V, et al. Risk factors for severe outcomes following 2009 influenza a (H1N1) infection: a global pooled analysis. PLoS Med. 2011;8:e1001053.
4. Morgan OW, Bramley A, Fowlkes A, et al. Morbid obesity as a risk factor for hospitalization and death due to2009 pandemic influenza $\mathrm{A}(\mathrm{H} 1 \mathrm{~N} 1)$ disease. PLoS ONE. 2010;5:e9694.

5. Sullivan SJ, Jacobson RM, Dowdle WR, Poland GA. 2009 H1N1 influenza. Mayo Clin Proc. 2010;85:64-76.

6. Cocoros NM, Lash TL, Demaria A, Klompas M. Obesity as a risk factor for severe influenza-like illness. Influenza Other Respir Viruses. 2013;8:25-32.

7. Coleman LA, Waring SC, Irving SA, Vandermause M, Shay DK, Belongia EA. Evaluation of obesity as an independent risk factor for medically attended laboratory-confirmed influenza. Influenza Other Respir Viruses. 2013;7:160-167.

8. Kwong JC, Campitelli MA, Rosella LC. Obesity and respiratory hospitalizations during influenza seasons in Ontario, Canada: a cohort study. Clin Infect Dis. 2011;53:413-421.

9. Sun Y, Wang Q, Yang G, Lin C, Zhang Y, Yang P. Weight and prognosis for influenza $A(\mathrm{H} 1 \mathrm{~N} 1)$ pdm09 infection during the pandemic period between 2009 and 2011: a systematic review of observational studies with meta-analysis. Infect Dis (Auckl). 2016;48:813-822.

10. Fiore A, Fry A, Shay D, Gubareva L, Bresee J, Uyeki T. Antiviral agents for the treatment and chemoprophylaxis of influenza recommendations of the advisory committee on immunization practices (ACIP). MMWR. 2011;60:1-25.

11. Aoki FY, Macleod MD, Paggiaro P, et al. Early administration of oral oseltamivir increases the benefits of influenza treatment. J Antimicrob Chemother. 2003;51:123-129.

12. Hiba V, Chowers M, Levi-Vinograd I, Rubinovitch B, Leibovici L, Paul $M$. Benefit of early treatment with oseltamivir in hospitalized patients with documented 2009 influenza A (H1N1): retrospective cohort study. J Antimicrob Chemother. 2011;66:1150-1155.

13. Ling LM, Chow AL, Lye DC, et al. Effects of early oseltamivir therapy on viral shedding in 2009 pandemic influenza $A$ (H1N1) virus infection. Clin Infect Dis. 2010;50:963-969.

14. Louie JK, Yang S, Acosta M, et al. Treatment with neuraminidase inhibitors for critically ill patients with influenza A (H1N1)pdm09. Clin Infect Dis. 2012;55:1198-1204.

15. Yu H, Feng Z, Uyeki TM, et al. Risk factors for severe illness with 2009 pandemic influenza a (H1N1) virus infection in China. Clin Infect Dis. 2011;52:457-465.

16. CDC. NPAO Data Trends and Maps-Location Summary-Michigan Location Summary. Center for Disease Control and Prevention. https://nccd.cdc.gov/NPAO_DTM/LocationSummary.aspx? state=Michigan. Accessed September 23, 2016.

17. Martin ET, Archer C, McRoberts J, et al. Epidemiology of severe influenza outcomes among adult patients with obesity in Detroit, Michigan, 2011. Influenza Other Respir Viruses. 2013;7:1004-1007.

18. Centers for Disease Control and Prevention (CDC). Behavioral Risk Factor Surveillance System Survey Data. 2011. https://www.cdc.gov/ brfss/questionnaires/. Accessed May 11, 2017.

19. Allard R, Leclerc P, Tremblay C, Tannenbaum T-N. Diabetes and the severity of pandemic influenza a (H1N1) infection. Diabetes Care. 2010;33:1491-1493.

20. Flu and People with Diabetes| Seasonal Influenza (Flu) |CDC. Center for Disease Control and Prevention. https://www.cdc.gov/flu/ diabetes/index.htm. Accessed July 21, 2017.

21. Chaves SS, Pérez A, Miller L, et al. Impact of prompt influenza antiviral treatment on extended care needs after influenza hospitalization among community-dwelling older adults. Clin Infect Dis. 2015;61:1807-1814.

22. Louie JK, Acosta M, Jamieson DJ, Honein MA. Severe 2009 H1N1 influenza in pregnant and postpartum women in california. $N$ Engl J Med. 2010;362:27-35.

23. Appiah GD, Chaves SS, Kirley PD, et al. Increased antiviral treatment among hospitalized children and adults with laboratory-Confirmed influenza, 2010-2015. Clin Infect Dis. November 2016:ciw745 DOI: 10.1093/cid/ciw745 
24. QuickFacts US Census. https://www.census.gov/quickfacts/table/ INC110215/2622000,00. Accessed February 27, 2017.

25. Capp R, Camp-Binford M, Sobolewski S, Bulmer S, Kelley L. Do adult medicaid enrollees prefer going to their primary care provider's clinic rather than emergency department (ED) for low acuity conditions? Med Care. 2015;53:530-533.

26. Sieck CJ, Hefner JL, Wexler R, Taylor CA, Mcalearney AS. Why do they do that?: Looking beyond typical reasons for non-urgent ED use among Medicaid patients. Patient Exp J. 2016;3:22-30.

How to cite this article: Segaloff HE, Evans R, Arshad S, et al. The impact of obesity and timely antiviral administration on severe influenza outcomes among hospitalized adults. J Med Virol. 2018;90:212-218.

https://doi.org/10.1002/jmv.24946 